\title{
A comparison of 4 different machine learning algorithms to predict lactoferrin content in bovine milk from mid-infrared spectra
}

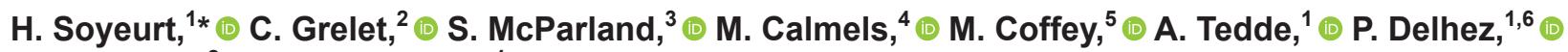 \\ F. Dehareng, ${ }^{2}$ (i) and N. Gengler ${ }^{1}$ (i) \\ ${ }^{1}$ TERRA research and teaching centre, Gembloux Agro-Bio Tech, University of Liège, Gembloux, Belgium \\ ${ }^{2}$ Valorisation of agricultural products, Walloon Research Centre, Gembloux, Belgium \\ ${ }^{3}$ Animal and Grassland Research and Innovation Centre, Teagasc, Moorepark, Fermoy, Co. Cork, Ireland \\ ${ }^{4}$ Research and development, Seenovia, Saint-Berthevin, France \\ 5 Livestock Breeding, Animal and Veterinary Sciences, Scotland's Rural College, Midlothian, UK \\ ${ }^{6}$ National fund for Scientific Research, Brussels, Belgium
}

\section{ABSTRACT}

Lactoferrin (LF) is a glycoprotein naturally present in milk. Its content varies throughout lactation, but also with mastitis; therefore it is a potential additional indicator of udder health beyond somatic cell count. Condequently, there is an interest in quantifying this biomolecule routinely. First prediction equations proposed in the literature to predict the content in milk using milk mid-infrared spectrometry were built using partial least square regression (PLSR) due to the limited size of the data set. Thanks to a large data set, the current study aimed to test 4 different machine learning algorithms using a large data set comprising 6,619 records collected across different herds, breeds, and countries. The first algorithm was a PLSR, as used in past investigations. The second and third algorithms used partial least square (PLS) factors combined with a linear and polynomial support vector regression (PLS + SVR). The fourth algorithm also used PLS factors, but included in an artificial neural network with 1 hidden layer (PLS + ANN). The training and validation sets comprised 5,541 and 836 records, respectively. Even if the calibration prediction performances were the best for PLS + polynomial SVR, their validation prediction performances were the worst. The 3 other algorithms had similar validation performances. Indeed, the validation root mean squared error (RMSE) ranged between 162.17 and $166.75 \mathrm{mg} / \mathrm{L}$ of milk. However, the lower standard deviation of cross-validation RMSE and the better normality of the residual distribution observed for PLS + ANN suggest that this modeling was more suitable to predict the LF content in milk

Received May 9, 2020.

Accepted August 10, 2020.

*Corresponding author: hsoyeurt@uliege.be from milk mid-infrared spectra $\left(\mathrm{R}^{2} \mathrm{v}=0.60\right.$ and validation $\mathrm{RMSE}=162.17 \mathrm{mg} / \mathrm{L}$ of milk). This PLS +ANN model was then applied to almost 6 million spectral records. The predicted LF showed the expected relationships with milk yield, somatic cell score, somatic cell count, and stage of lactation. The model tended to underestimate high LF values (higher than $600 \mathrm{mg} / \mathrm{L}$ of milk). However, if the prediction threshold was set to $500 \mathrm{mg} / \mathrm{L}, 82 \%$ of samples from the validation having a content of LF higher than $600 \mathrm{mg} / \mathrm{L}$ were detected. Future research should aim to increase the number of those extremely high LF records in the calibration set. Key words: milk, lactoferrin, mid infrared, machine learning

\section{INTRODUCTION}

Lactoferrin ( $\mathbf{L F})$, an $80-\mathrm{kDa}$ glycoprotein naturally present in milk, is synthetized by the mammary gland epithelial cells (Molenaar et al., 1996) and has antibacterial, antiviral, and antifungal activities that can potentially improve a cow's disease resistance. More details about the nutraceutical and pharmaceutical properties of milk bovine LF can be found in the review of Giansanti et al. (2016). These immune effects explain why the synthesis of LF increases in the presence of mastitis infection (Gaunt et al., 1980; Hagiwara et al., 2003), but the responses can be different following the incriminated pathogens (Kawai et al., 1999; Chaneton et al., 2008). Moreover, the content of LF in milk can also vary naturally depending on parity, age, lactation stage (Gaunt et al., 1980; Hagiwara et al., 2003), and breed (Król et al., 2010). Consequently, there is an interest in quantifying the LF content in milk at the individual level for different issues related to animal welfare (i.e., early detection of infection) and human health due to the presence of this active biomolecule in milk. Indeed, humans can also profit from LF by taking 
it orally due to its anti-infective, anti-cancer, and antiinflammatory properties (Wakabayashi et al., 2006).

Content of LF can be quantified using an immunodiffusion method (Hagiwara et al., 2003), but its quantification is more often based on ELISA (Chen and Mao, 2004; Chaneton et al., 2013). Unfortunately, those methods are too labor intensive for routine screening of the cow population at the individual scale, as desired. Therefore, alternative methods offering a quantification at a low cost must be found. In 2007, a first study was published about the prediction of LF content in milk using milk mid-infrared (MIR) spectrometry (Soyeurt et al., 2007), a technology largely implemented in most milk laboratories. This first study measured the content of LF in a limited number of samples (i.e., 69 records). However, this first study and a follow-up study based on a bigger data set (i.e., 2,499 records; Soyeurt et al., 2012) validated the potential of MIR to provide a relevant indicator of LF. Indeed, the cross- and external-validation coefficients of determination $\left(\mathbf{R}^{2}\right)$ and root mean square error (RMSE) obtained from this second study were of 0.71 and 0.60 , and of 50.55 and $58.98 \mathrm{mg} / \mathrm{L}$ of milk, respectively. Recently, the European Milk Recording network (www.milkrecording .eu) has developed its own equation from more than 2,000 records and offers the prediction service of this biomolecule to its members. By combining all of those data sets, new perspectives are shown by trying different machine learning algorithms, which could improve the current accuracy of LF prediction. Indeed, all LF prediction equations were built using partial least squares regressions (PLSR).

Since the nineties, several pieces of research were conducted in dairy science using an artificial neural network (ANN); for instance, ANN have been used to analyze breeding dairy patterns (Finn et al., 1996) or to predict the incidence of clinical mastitis (Yang et al., 2000) or milk yield (Grzesiak et al., 2006). To our knowledge, only 3 articles mainly or partly used the milk MIR spectra as predictors. Those studies were concerned with the prediction of conception success for a given insemination (Hempstalk et al., 2015), content of blood BHB (Pralle et al., 2018), and feed intake (Dórea et al., 2018). Several reasons could potentially explain the large use of PLSR to model milk MIR spectral data. Absorbance values of consecutive spectral data points are highly correlated. Therefore, collinearity problems are present if conventional simple linear regression is used with all spectral data points as explanatory variables. Fortunately, some solutions exist to solve this problem. The first one consists of selecting a limited number of spectral points by trying to keep the most relevant information in the data set, while limiting the correlations between them. Then, those low correlated spectral points can be included in a multivariate regression. The second possibility is to reduce the dimensionality of the spectral $\mathrm{X}$ matrix by using a principal component analysis (PCA). The PCA latent variable $(\mathbf{L V})$ can then be used in a multivariate regression, commonly named principal component regression. However, the PCA methodology used to define those LV considers only the spectral variability and not the variability of the trait to be predicted. This could lead to a lack of relevant spectral information to predict the trait of interest. The partial least square (PLS) method solves this problem by defining LV by simultaneously considering the variabilities of $\mathrm{X}$ and $\mathrm{Y}$ (Despagne et al., 2000). This explains why this methodology was, and still is, mainly used to develop milk MIR models to predict traits related to milk quality, such as fatty acids (Soyeurt et al., 2011), cheese making properties (De Marchi et al., 2009), BW (Soyeurt et al., 2019), fertility (Delhez et al., 2020), traits related to animal welfare (Grelet et al., 2016), and environmental issues (Vanlierde et al., 2016). Unfortunately, PLS is able to consider weakly nonlinear relationships by adding LV, potentially leading to over-fit the developed prediction model (Thissen et al., 2004). Other machine learning algorithms such as support vector regression (SVR) and ANN have the ability to model the nonlinear relations (Thissen et al., 2004). Compared with ANN, SVR can deal with a high number of input variables (Thissen et al., 2004). However, after a feature selection, ANN can be also efficient. Moreover, using weights obtained from a past calibration data set, the weights used in an ANN network can be updated using a new calibration data set. This is particularly interesting when a many phenotypes that are useful to predict the trait of interest are available.

The computational methodology differs between SVR and ANN. Support vector regression was created by Vapnik (Thissen et al., 2004) and defines a classification boundary between records to minimize the distance between the records and the boundary by considering a certain limit of detection (called epsilon). More specifically, SVR is a method that selects a reduced number of samples, the support vectors, which define the best sparse deterministic regression relationship between the MIR data and the reference values. To ensure a global solution, a penalty (called C penalty) is used during the computation. Different kernels can be used to compute the boundary, such as linear, polynomial, or radial kernel; each kernel has its own parameters to be optimized. More details about this method are given by Thissen et al. (2004). The ANN, initially introduced by McCulloch and Pitts (1943), is the basis of deep learning that tries 
to mimic neuronal brain activity (i.e., the computer learns by experience). An ANN is composed of different layers including units: 1 input layer, 1 output layer and a certain number of hidden layers. The number of hidden layers and its corresponding units must be defined by the user. A higher number of hidden layers and their corresponding units yields a higher complexity of the model, and consequently a higher potential to over-fit the prediction model. The ANN algorithm aims to estimate the weights of each relation among units by using, for instance, the back-propagation methodology. The cost function is related to the minimization of the residual error. The ANN model will provide a response of 1 or more variables when given many explanatory variables (i.e., units; Beck, 2018). Therefore, SVR and ANN require the optimization of several parameters. To achieve this objective and to get a global solution, it is important to have a large data set, but the size of the data set is often limited and potentially explains why those methods are not often used in milk MIR spectrometry . Compared with PLSR, however, SVR and ANN in themselves do not solve the issue of collinearity between spectral data points. Consequently, there is an interest to combine the dimension reduction obtained by PLS with SVR or ANN algorithms. Therefore, the objective of the current study was to compare the accuracy of predictions of milk LF content from milk MIR spectra using 4 different machine learning algorithms: PLSR, linear and polynomial SVR coupled with PLS LV, and ANN coupled with PLS LV.

\section{MATERIALS AND METHODS}

All analyses were performed using $\mathrm{R}$ software (version 4.0.1; https://www.r-project.org/).

\section{Data}

The first data set comprised 3,965 milk samples $(50 \%$ of morning and $50 \%$ of evening milk) preserved with bronopol and collected between April 2005 and April 2006 in Belgium, between April 2009 and August 2009 in Ireland, and during August 2009 in Scotland. Part of those samples was also used in a previous study (Soyeurt et al., 2012). The Belgian samples $(\mathrm{n}=549)$ were analyzed using one MilkoScan FT6000 spectrometer (Foss, Hillerod, Denmark) located in the milk laboratory "Comité du Lait" (Battice, Belgium). The Irish and Scottish samples $(\mathrm{n}=3,416)$ were also analyzed on the same brand of spectrometer at the Animal and Grassland Reasearch and Innovation Centre, Teagasc Moorepark (Fermoy, Co. Cork, Ireland). The spectral data of each sample contained 1,060 wavenumbers. The second data set comprised 2,654 milk samples (50\% of morning milk and $50 \%$ of evening milk) collected by the European Milk Recording network in France ( $\mathrm{n}=$ $1,333)$, Luxembourg $(\mathrm{n}=246)$, England $(\mathrm{n}=500)$, and Germany $(\mathrm{n}=575)$ between June 2016 and January 2017. The samples in this second data set were selected based on their LF content predicted using the equation developed by Soyeurt et al. (2012) to increase the variability over what was present in the samples of the first data set. All samples were analyzed using either FT+ MilkoScan spectrometers (Foss, Hillerod, Denmark) or Bentley spectrometers (Chaska, MN). The spectral data were then standardized based on the procedure explained by Grelet et al. (2017). All aspects related to this standardization were managed by the European Milk Recording network and resulted in all samples having 1,060 harmonized wavenumbers and absorbance values available for analysis. A single milk sample per cow was selected from animals of different breeds across several herds and countries right across lactation.

\section{Lactoferrin Quantification}

Lactoferrin concentration was quantified from the milk samples already analyzed by infrared spectroscopy using commercial ELISA kits: Bovine Lactoferrin ELISA Quantification kit from Bethyl Laboratories Inc. (Montgomery, TX) for the first data set and $\mathrm{e}_{-}$ bLF_01 kit from IDBiotech (Issoire, France) for the second data set. The Belgian samples were analyzed by Gembloux Agro-Bio Tech - University of Liège (Gembloux, Belgium). The ELISA analyses of the Irish and Scottish samples were conducted by Enfer Laboratories (Naas Co. Kildare, Ireland). The ELISA analysis of the second data set was conducted at Seenovia (SaintBerthevin, France). The samples were diluted 1:1,000; $1: 2000 ; 1: 4,000 ; 1: 6,000 ; 1: 8,000$, or $1: 10,000$ in sample buffer. The LF concentrations used for the calibration were the average of at least 2 ELISA measures taken on the same milk sample.

\section{Spectral Pretreatment}

The spectral data coming from the first data set were not standardized, as this procedure did not exist when the samples were collected. Therefore, to correct for a potential baseline drift, the first derivative was applied to the recorded spectra for the data set 1 and standardized spectra for the data set 2 using the following formula:

$$
\text { wavenumber }_{i}=\text { wavenumber }_{i}-\text { wavenumber }_{i+g a p},
$$


where wavenumber $_{i}{ }_{i}$ represents the first derivative value of the ith wavenumber, wavenumber $r_{i}$ is the raw value observed for the $i$ th wavenumber, and the gap is the window chosen for the derivation and was equal to 5 . Then, the wavenumbers located in the most informative regions were selected. Thus, a total of 277 wavenumbers were kept for this study and were located from 950 to $1,580 \mathrm{~cm}^{-1}$, from 1,720 to $1,770 \mathrm{~cm}^{-1}$, from 1,780 to $1,850 \mathrm{~cm}^{-1}$, and from 2,800 to $2,970 \mathrm{~cm}^{-1}$.

The presence of potential spectral outliers was assessed by estimating the standardized Mahalanobis (Whitfield et al., 1987) distance (also called GH distance) for all recorded spectra. To allow the inversion of the matrix needed to calculate the Mahalanobis distance due to the high collinearity existing between some spectral points, a PCA was performed using FactoMineR package (version 1.42; Lê et al., 2008), defining 22 uncorrelated principal components (PC). This explained $99.04 \%$ of the spectral variability. The formula used to calculate the $G H$ distance was as follows:

$$
G H=\frac{\sqrt{(x-\mu)^{T} S^{-1}(x-\mu)}}{n P C},
$$

where $x$ is the PC score of a specific spectrum; $\mu$ is the mean of PC scores estimated from the entire data set; $S$ corresponds to the (co)variance matrix between PC scores estimated from the entire data set; $n P C$ is the number of $\mathrm{PC}$ used in the calculation (i.e., 22 in our case). The PC analysis was performed on a combined data set containing all of the records to improve the certainty of spectral outlier detection. A total of 86 records with a $G H$ higher than 5 were discarded from the data set. The final cleaned data set contained 6,533 records (i.e., 3,931 and 2,602 records of the first and second data sets, respectively).

\section{Data Splitting}

To perform a complete external validation, the data coming from 2 different DHI organizations ( 1 in Germany and 1 in England) were not used to calibrate the model. This external-validation data set represented 836 samples. The remaining samples $(\mathrm{n}=5,541)$ were used to calibrate the model and performed 2 different cross-validations: 10-fold cross-validation where the samples were chosen randomly in the calibration data set and a leave-one DHI out cross-validation. The leave-one DHI out cross-validation allows to evaluate the models with samples coming from the same context (countries, diets, breeds) as mentioned by Prekopcsak et al. (2010). We supposed that the first data set contained $9 \mathrm{DHI}$, which corresponded to 9 herds in this case. The second data set contained records coming from 6 different DHI, but records coming from 2 DHI were kept for the external validation as explained previously. Therefore, the leave-one DHI out cross-validation procedure considered 13 groups. Two cross-validations were tested and compared in this study as a random N-fold cross-validation could provide over-optimistic prediction performances (Wang and Bovenhuis, 2019).

\section{Machine Learning Algorithms}

All machine learning algorithms used in this study used the CARET package version 6.0-86 (Kuhn, 2008). For all models, the spectral data were scaled and centered before computation.

A PLSR was performed on the 277 selected wavenumbers, using the method = "pls" as an argument in the train function of CARET package. The maximum number of PLS latent variables was set to 50 . The optimized number of factors was chosen using the selectionFunction = "oneSE" and "best" as arguments in the train function of CARET package. The "best" selection function defines the optimal model as the one having the lowest cross-validation RMSE (RMSEcv). The "oneSE" selection function allows for the selection of a simpler model with a lower RMSE within 1 standard error of the lowest obtained RMSEcv. This simpler model is assumed to have a better generalization.

The computation of SVR was based on linear and polynomial kernels and was implemented using the method = "svmLinear" or "svmPoly" as arguments in the train function of the CARET package. For both kernels, the expand.grid function was used to test different values to optimize the required parameters. For "svmLinear," the tested $\mathrm{C}$ values were $0.01,0.05,0.1$, $0.25,0.5,0.75,1,1.25,1.5,1.75,2$, and 5 . For the "svmPoly" kernel, the tested values were $0.25,0.5,1$ and 2 for C; 1 until 3 for the polynomial degree; and 0,001, $0.01,0.1$ and 1 for the scale. For both kernels, the epsilon parameter was set to 0.1. As for PLSR, the optimal parameters were chosen using the selection function "best" or "oneSE." A total of 26 PLS factors explaining $99 \%$ of the spectral variability were combined in SVR to limit the problem of overfitting. The interest in using PLS factors instead of other more conventional selections of features is based on the fact that PLS will extract factors by considering simultaneously the spectral variability and the variability of the trait to be predicted.

An ANN seems to be more powerful when the selection of features is made before the modeling (Thissen et 
al., 2004). Thus, as performed for SVR, ANN included the 26 PLS latent variables instead of the 277 initially selected wavenumbers. We tested an ANN based on 1-layer perceptron in this study. This ANN architecture is composed of a hidden layer to minimize the risk of overfitting. To estimate the weights related to this ANN design, a back propagation was used. This model was performed using method = "nnet" as argument in the train function of the CARET package. Different numbers of units in the unique hidden layer (ranging from 1-5) were tested using the expand.grid function. To ensure a global solution, a penalty was introduced during the computation of weights (called decay). Decay values of $0,0.0001,0.001,0.01,0.1,0.2,0.3,0.4$, and 0.5 were tested using the expand.grid function. As done for other algorithms, the optimized values for the size and decay were chosen using the selection function "best" or "oneSE." The maximum iteration for the weight estimations was set to 1,000 to be sure to reach the convergence.

The prediction performances of the different models developed were assessed by estimating the calibration, 10-fold cross-validation, leave-one DHI out cross-validation, and external-validation coefficients of determination $\left(\mathbf{R}^{2} \mathbf{c}, \mathbf{1 0}\right.$-fold $\mathbf{R}^{2} \mathbf{c v}$, DHI $\mathbf{R}^{2} \mathbf{c v}$, and $\mathbf{R}^{2} \mathbf{v}$, respectively) as well as their corresponding RMSE (i.e., RMSEc, 10-fold RMSEcv, DHI RMSEcv, and RMSEv, respectively). Distributions of residuals were also studied for all models.

\section{Prediction of LF from the Walloon Milk Recording Database}

As the calibration and validation sets were composed of a limited number of records and were not representative of the studied dairy population due to the sampling procedure used, there was an interest in observing the behavior of the prediction on a large-scale spectral database. Indeed, this allowed observing the behavior of the predictions according to known sources of variation such as the stage of lactation, parity, breed, and season. Therefore, the machine learning algorithm chosen as the best based on the validation prediction performances was applied on the first derived milk MIR spectra. The spectral database is managed by the Walloon Breeding Association (Awé, Ciney, Belgium). This database is related to the milk recording. A total of 5,651,470 records were collected between January 2007 and March 2020 from 349,396 cows in 1,963 herds. The average values for the predicted LF were estimated according to the stage of lactation, the milk yield, and the SCS $[\log 2(\mathrm{SCC} / 100,000)+3]$. The correlations between the predicted LF and milk yield, fat and protein contents, as well as SCC and SCS were also estimated.

\section{RESULTS AND DISCUSSION}

\section{Descriptive Statistics and Data Cleaning}

The LF content measured in the samples included in the first data set $(\mathrm{n}=3,931)$ ranged from 3 to 2,038 $\mathrm{mg} / \mathrm{L}$ of milk, with an average of $202 \pm 170 \mathrm{mg} / \mathrm{L}$ of milk. The LF content in the second data set $(\mathrm{n}=2,602)$ varied from 6 to $1,299 \mathrm{mg} / \mathrm{L}$ of milk, with an average of $325 \pm 257 \mathrm{mg} / \mathrm{L}$ of milk. The average content observed in the first data set was within the expected range compared with other published articles. For instance, Gaunt et al. (1980) found an average content of LF of $266 \pm$ $136 \mathrm{mg} / \mathrm{L}$ of milk from a first set of 4 herds, and $228 \mathrm{mg}$ $\pm 112 \mathrm{mg} / \mathrm{L}$ of milk from a second set of 4 herds. Cheng et al. (2008) found a slightly lower content of LF in bovine milk $(177 \pm 120 \mathrm{mg} / \mathrm{L})$ from samples collected on cows without mastitis infection. In a previous article using a part of the first data set $(n=2,499)$, Soyeurt et al. (2012) found an average content of LF equal to $163 \pm 103 \mathrm{mg} / \mathrm{L}$ of milk. The content observed in the second data set seemed to be high compared with the literature. Moreover, the standard deviation (SD) was also higher compared with the first data set. This can be related to the sample selection. Indeed, the samples included in the second data set did not come from entire herds as they were selected based on a past LF MIR predictive model to cover as much as possible of the LF content and spectral variation. However, the ranges of variation observed in both data sets were very high, with extremely high LF measurements. This could be related to the fact that some samples could be collected from cows having subclinical mastitis. Some authors found a positive relationship between the content of LF and the presence of mastitis (Kawai et al., 1999) even if the response differed following the incriminated pathogens (Chaneton et al., 2008). For instance, Gaunt et al. (1980) measured average LF content of $222 \pm 168$ $\mathrm{mg} / \mathrm{L}$ of milk for healthy cows to $640 \pm 250 \mathrm{mg} / \mathrm{L}$ of milk for cows presenting mastitis. Cheng et al. (2008) obtained similar values (i.e., $742 \pm 374 \mathrm{mg} / \mathrm{L}$ of milk for cows suspected of having mastitis on the basis of the SCC of the milk). The distribution of LF (Figure 1) and spectra (data not shown) from the 2 data sets were complementary. This was expected because samples of the data set 2 were selected to complement the data set 1 .

\section{Lactoferrin Predictions Using Milk MIR Spectrometry}

Two kinds of cross-validation procedures were tested in this study to fix the model parameters (i.e., number of LV for PLS regressions; C value for linear SVR; C, scale, and degree for polynomial SVR; and size and de- 
cay for ANN). The leave-one DHI out cross-validation leads to the model being under-fit, resulting a higher prediction error. Indeed, RMSEv values were higher than $175 \mathrm{mg} / \mathrm{L}$ of milk and were always greater for models developed using the leave-one DHI out crossvalidation when compared with models built using the 10-fold cross-validation (Table 1). Moreover, the high RMSEcv SD for models built using the leave-one DHI out cross-validation (i.e., $>90 \mathrm{mg} / \mathrm{L}$ of milk) confirmed the low robustness of the developed models. This suggests that too many informative samples were taken out from the calibration set. For instance, during a 10 -fold cross-validation, samples coming from the same herd can be in the training and validation set involving relevant information to provide a better prediction. Consequently, the use of a 10 -fold cross-validation to parametrize a model is still relevant to limit the underfitting; however, as mentioned by Wang and Bovenhuis (2019), this procedure leads to over-optimistic prediction performances. Indeed, the observed RMSEcv were always lower than the one observed for the validation set (Table 1 ). The $\mathrm{R}^{2} \mathrm{cv}$ values obtained from models developed using 10-fold cross-validation were similar between models used and ranged from 0.51 to 0.56 . Similarly, the observed RMSEcv were also globally the same and ranged from 138.40 to $144.60 \mathrm{mg} / \mathrm{L}$ (Table 1). This suggested similar prediction performances. However, RMSEcv SD was higher for PLS + polynomial SVR compared with other tested algorithms. Based on the external validation, PLSR, PLS + polynomial SVR and PLS + ANN showed similar validation prediction performances with respective RMSE values of 163.76, 166.75 , and $162.17 \mathrm{mg} / \mathrm{L}$ of milk. However, the correlation values between predictions on the validation set (n $=836$ ) suggested some differences. Higher correlations were observed between the predictions given by the PLSR and PLS + linear SVR models (0.99) compared with PLS + polynomial SVR or PLS + ANN (0.95 for both algorithms). The correlation between PLS + ANN and PLS + polynomial SVR was 0.94. From Figure 2, it is clear that the relationships between the predictions made from PLSR and PLS + linear SVR models is strong. However, the relationship of those models with other tested ones was not linear. There appears to be
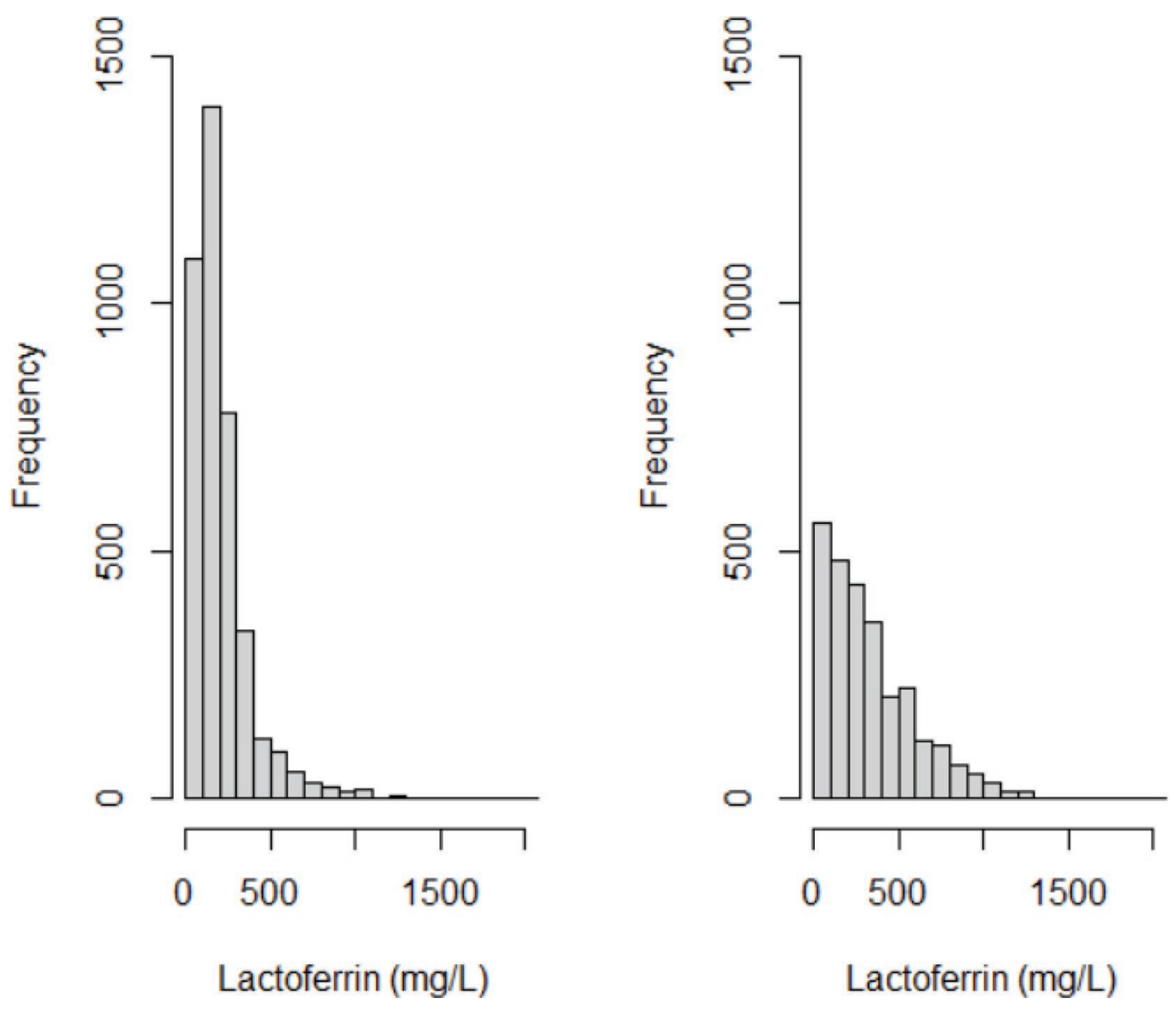

Figure 1. Distribution of ELISA lactoferrin quantifications in the first data set (on the left) and the second data set (on the right). 
Table 1. The 10-fold cross-validation and external-validation performances for predicting lactoferrin content in milk using 4 different machine learning algorithms ${ }^{1}$

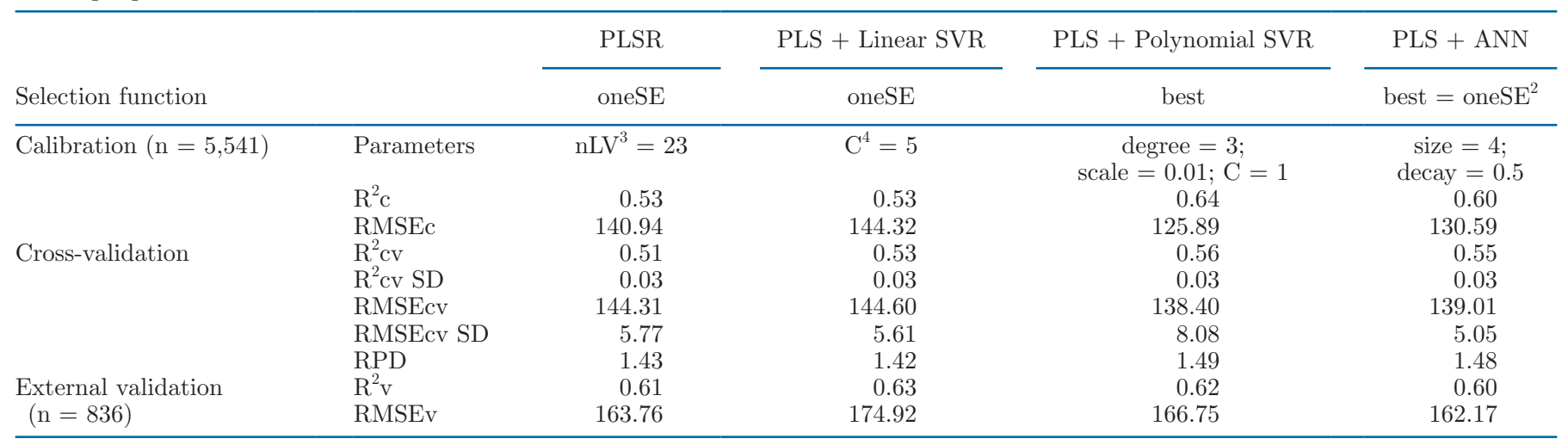

${ }^{1}$ PLSR $=$ partial least squares regression; PLS + Linear SVR = linear support vector regression (SVR) based on 26 PLS latent variables; PLS + Polynomial SVR = linear SVR based on 26 PLS latent variables; PLS + ANN = modeling based on artificial neural network including 26 PLS latent variables in the input layer and one hidden layer. degree $=$ the degree of polynomials used in the kernel; scale $=$ the scaling parameter of the polynomial; size $=$ the number of nodes in the hidden layer; decay $=$ the penalty used for $\mathrm{ANN}$. $\mathrm{R}^{2} \mathrm{c}=$ calibration coefficient of determination $\left(\mathrm{R}^{2}\right)$ : RMSEc $=$ calibration root mean square error $(\mathrm{RMSE}) ; \mathrm{R}^{2} \mathrm{cv}=$ cross-validation $\mathrm{R}^{2} ; \mathrm{R}^{2} \mathrm{cv} \mathrm{SD}=$ standard deviation of $\mathrm{R}^{2} \mathrm{cv}$; $\mathrm{RMSE} \mathrm{cv}$ $=$ cross-validation RMSE; RMSEcv SD = standard deviation of RMSEcv; RPD = the standard deviation divided by RMSEcv; $\mathrm{R}^{2} \mathrm{v}=\mathrm{validation}^{2}$ $\mathrm{R} 2 ; \mathrm{RMSEv}=$ validation RMSE.

${ }^{2}$ The selection function "best" and "oneSE" provided the same results.

${ }^{3} \mathrm{nLV}=$ number of PLS latent variables.

${ }^{4} \mathrm{C}=$ cost penalty used for SVR.

a saturation for low and high prediction values (i.e., S shape).

Therefore, even though the predictions made by the 4 models were highly correlated (i.e., higher than 0.94), the low and high values behaved differently. Moreover, the range of predictions was really different, with PLS + linear SVM and PLS + ANN having a reduced range compared with PLSR and PLS + polynomial SVR. Moreover, except for PLS + ANN, all other tested algorithms had the tendency to predict negative values (Table 2). The correlation between residuals and predicted content of LF ranged from 0.64 for PLS + ANN to 0.77 for PLS + linear SVM based on the training set. From the validation set, these correlation values were between 0.60 for PLS + ANN and 0.83 for PLS + linear SVM. These correlation values were lower with the squared residuals (from 0.49 for PLS + ANN to 0.61 for PLS + linear SVR and from 0.30 for PLS + ANN to 0.68 for PLS + linear SVR based on the training and validation sets, respectively). This suggested that higher errors were made for samples having a high content of LF. The validation prediction performances, the robustness (low RMSEcv SD; Table 1), the prediction of positive values (Table 2), and the lowest correlation between squared residuals and LF content observed for PLS + ANN suggested that this modeling is the most relevant to predict daily LF content in milk from milk MIR spectrometry. For another application dedicated to dairy science, Dórea et al. (2018) also obtained better prediction perfor- mances using ANN, including a hidden layer after a selection of input variables compared with PLSR to predict feed intake. Pralle et al. (2018) obtained similar performances for PLSR and ANN, also including a hidden layer to predict blood BHB.

Compared with the previous studies published by our team on the same topic, the prediction error observed in the current study was higher than what we observed in the past $[\mathrm{RMSEv}=77.26 \mathrm{mg} / \mathrm{L}$ of milk in Soyeurt et al. (2012) vs. $163.76 \mathrm{mg} / \mathrm{L}$ in the current study based on PLSR or $162.17 \mathrm{mg} / \mathrm{L}$ of milk for PLS + ANN]. However, this is difficult to compare because the validation data set was not the same, and all spectra were not standardized. If the equation published earlier (Soyeurt et al., 2012) is applied on the current validation set, the validation prediction error is $462 \mathrm{mg} / \mathrm{dL}$ with a $R^{2} \mathrm{v}$ equal to 0.02. Even if this old equation was built from a part of data set 1 (2,499 samples), the variability in the current data sets is higher, and the past prediction equation is not suitable to predict those records. This could also be related to the fact that 2 different ELISA kits were used. However, the residual distributions obtained after using the PLS + ANN model were similar for data sets 1 and 2 (data not shown). Moreover, an ANOVA also confirmed that the differences between residuals observed from data sets 1 and 2 were not significant. It is interesting to note that $50 \%$ of records had a residual error between -69.06 and $51.66 \mathrm{mg} / \mathrm{L}$ of milk and between -85.78 and $91.37 \mathrm{mg} / \mathrm{L}$ of milk for the training and validation set, respectively. 
$-200 \quad 400 \quad 800$
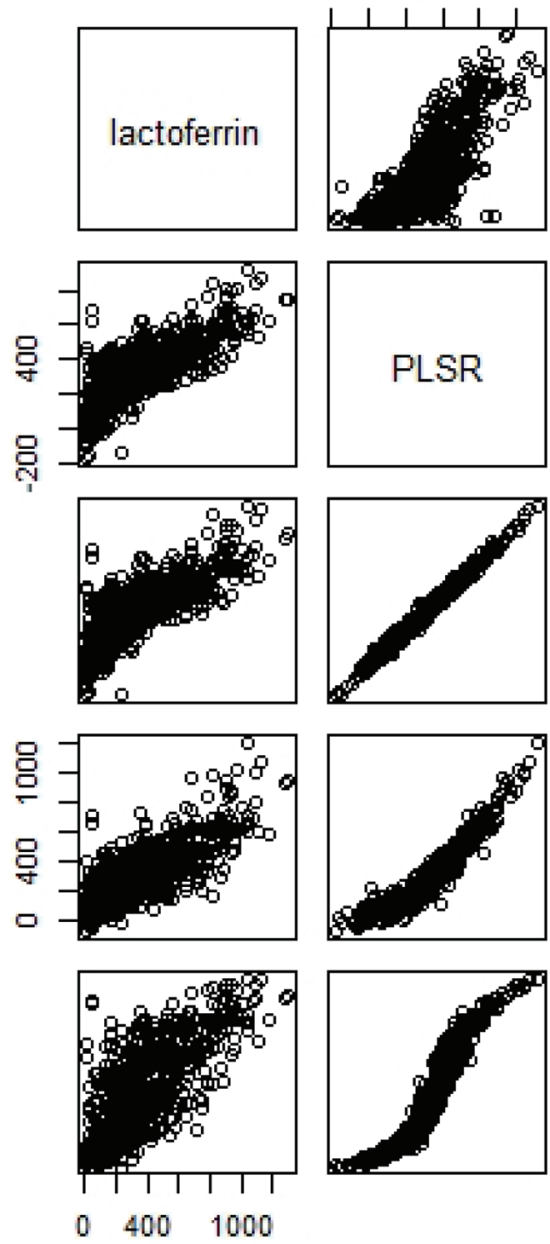
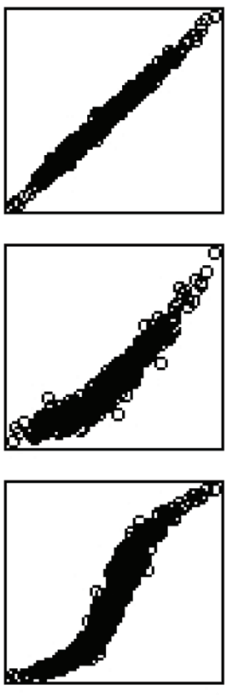
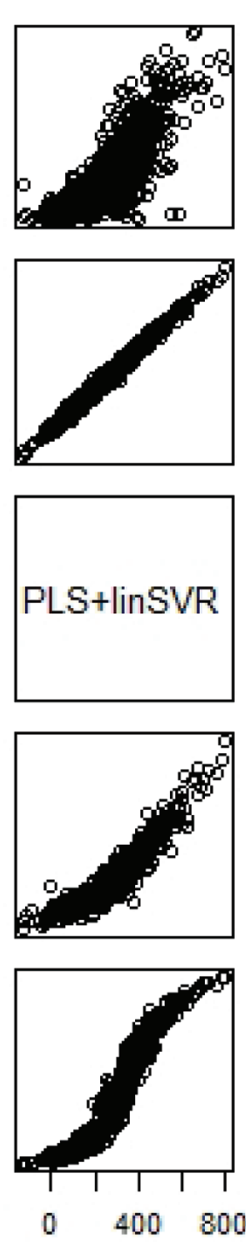

$0 \quad 400 \quad 1000$
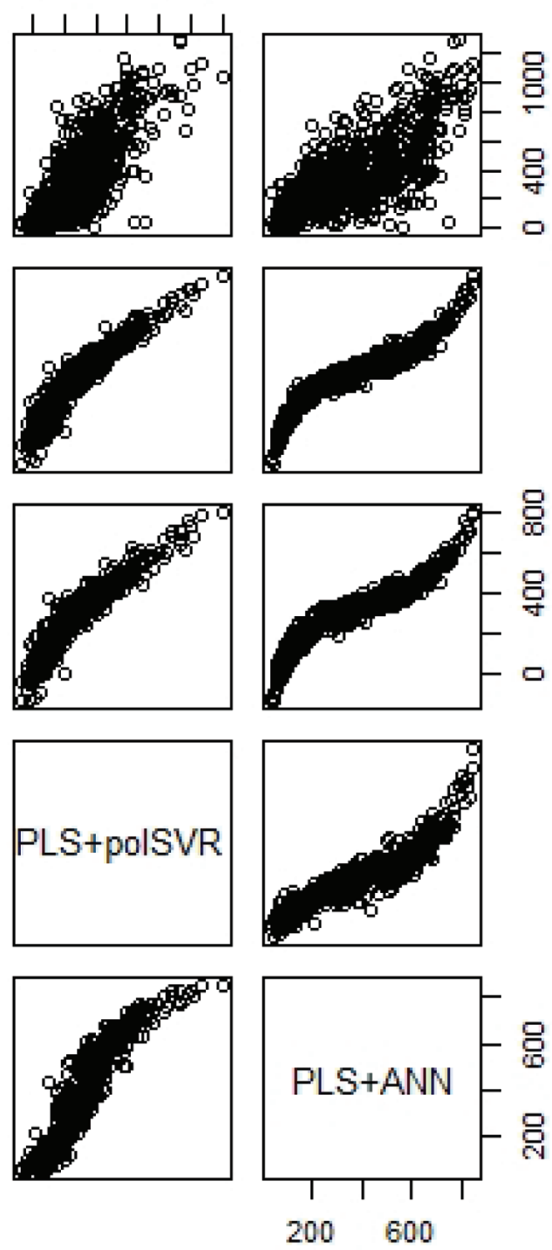

Figure 2. Relationships between reference lactoferrin content ( $\mathrm{mg} / \mathrm{L}$ of milk) and the predictions obtained using 4 different machine learning approaches applied on the validation set. PLSR $=$ partial least squares regression; PLS+linSVR $=26$ PLS factors included in a linear support vector regression $(\mathrm{SVR}) ; \mathrm{PLS}+$ polSVR $=26 \mathrm{PLS}$ factors included in a polynomial SVR; PLS+ANN $=26$ PLS factors included in an artificial neural network having a hidden layer.

Table 2. Data distribution of the reference lactoferrin contents as well as the predictions obtained from the developed models after a 10-fold cross-validation

\begin{tabular}{|c|c|c|c|c|c|c|c|c|c|}
\hline Item $^{1}$ & \multicolumn{9}{|c|}{ Data distribution (mg/L of milk) } \\
\hline \multicolumn{10}{|l|}{ Training set } \\
\hline PLSR & -277.95 & 35.10 & 77.14 & 142.16 & 218.59 & 308.55 & 430.42 & 521.86 & $1,244.38$ \\
\hline PLS + linSVR & -195.05 & 37.97 & 74.54 & 128.68 & 194.55 & 271.75 & 385.47 & 466.19 & $1,060.68$ \\
\hline PLS + polSVR & -47.40 & 51.77 & 74.18 & 119.48 & 187.15 & 271.52 & 413.47 & 532.49 & $1,423.58$ \\
\hline PLSR & -162.60 & 13.98 & 70.29 & 165.56 & 320.69 & 420.68 & 518.84 & 591.00 & 912.53 \\
\hline PLS + linSVR & -129.09 & 22.84 & 68.17 & 150.40 & 290.59 & 367.41 & 455.30 & 520.24 & 795.47 \\
\hline PLS + polSVR & -72.59 & 27.30 & 55.13 & 123.53 & 263.11 & 381.74 & 533.62 & 626.71 & $1,200.00$ \\
\hline $\mathrm{PLS}+\mathrm{ANN}$ & 36.67 & 67.71 & 85.11 & 127.48 & 313.83 & 483.87 & 633.11 & 682.23 & 851.38 \\
\hline
\end{tabular}

${ }^{1} \mathrm{PLSR}=$ partial least squares regression; PLS + linSVR $=$ linear support vector regression $(\mathrm{SVR})$ based on 26 PLS latent variables; PLS + polSVR = polynomial SVR based on 26 PLS latent variables; PLS + ANN = modeling based on an artificial neural network including 26 PLS latent variables in the input layer and a hidden layer. 


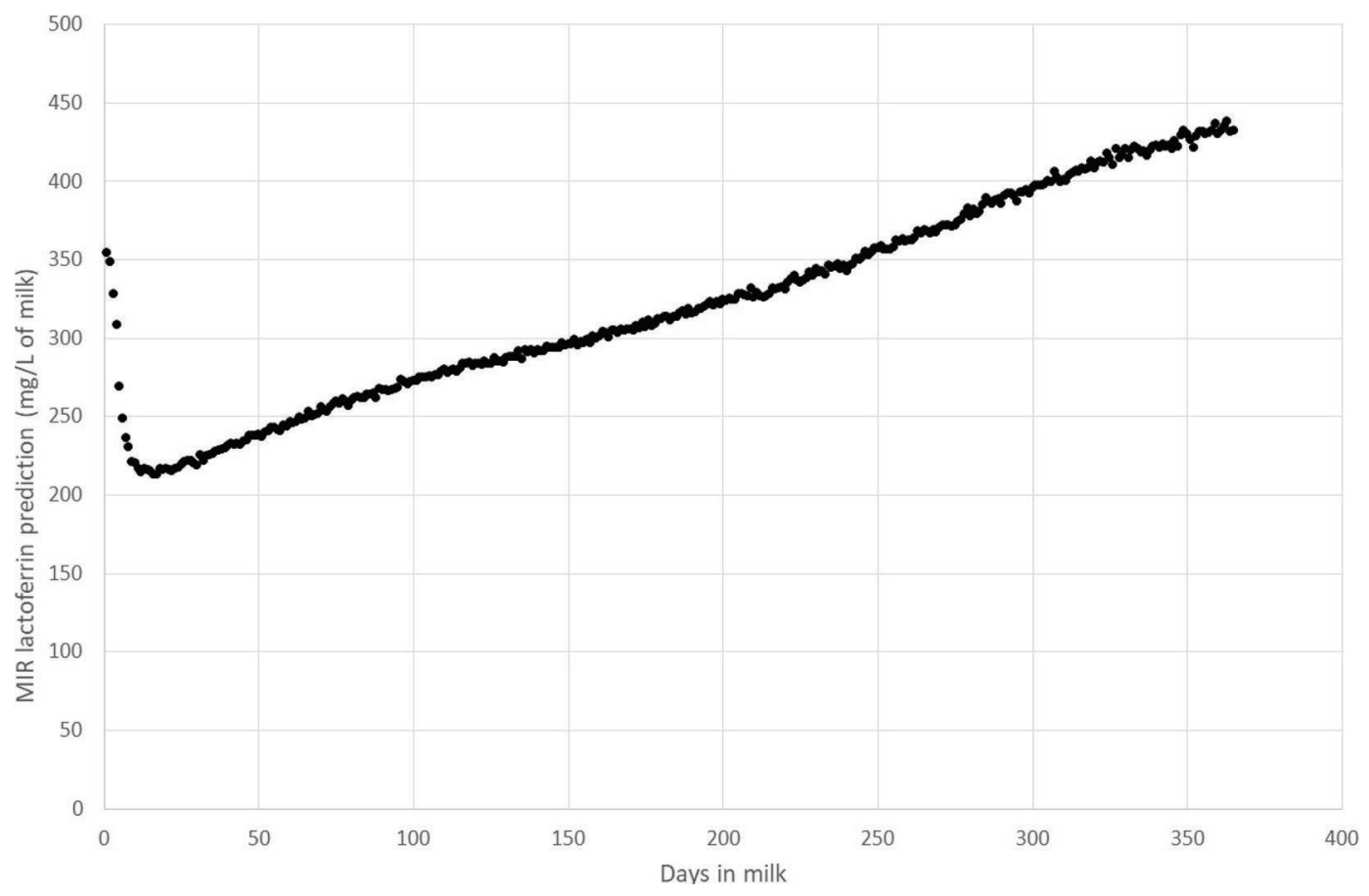

Figure 3. Evolution of lactoferrin content predicted by mid-infrared spectrometry following the stage of lactation.

In addition to the interest in predicting the quantity of LF in milk, it could be useful to know if the models were able to detect extreme values. Indeed, even if a high prediction error exists for the records having a high content of $\mathrm{LF}$, the prediction increased as expected, but with a lower intensity. The PLS + ANN model gave predictions allowing detection of $65 \%$ of the records with a content of LF higher than $600 \mathrm{mg} / \mathrm{L}$ of milk in the training set if we fixed the prediction limit to $500 \mathrm{mg} / \mathrm{L}$ of milk. This proportion reached up to $82 \%$ for the samples in the validation set. The threshold of $600 \mathrm{mg} / \mathrm{L}$ was used as the RMSE started to relate to the content of LF from this content and because authors including Gaunt et al. (1980) and Kawai et al. (1999) mentioned that a such high content is potentially related to cows having mastitis. Due to the distribution of LF observed in Figure 1, we have also tested the log-transformation, but the results were not better (data not shown).

\section{PLS + ANN Model Applied to a Large Spectral Database}

The PLS + ANN model was applied to 5,651,470 records from cows within the first $365 \mathrm{~d}$ in milk. The obtained average prediction was $307.80 \mathrm{mg} / \mathrm{L}$ of milk with a SD of $209.17 \mathrm{mg} / \mathrm{L}$ of milk. The minimum and maximum values were 19.74 and $1,121.00 \mathrm{mg} / \mathrm{L}$, respectively. As observed on the training and validation data sets, no negative predictions were observed.

The LF content predicted using MIR varied according to the stage of lactation (Figure 3 ). This variation

Table 3. Evolution of lactoferrin content predicted by mid-infrared (MIR) spectrometry following the stage of lactation and comparison with the literature

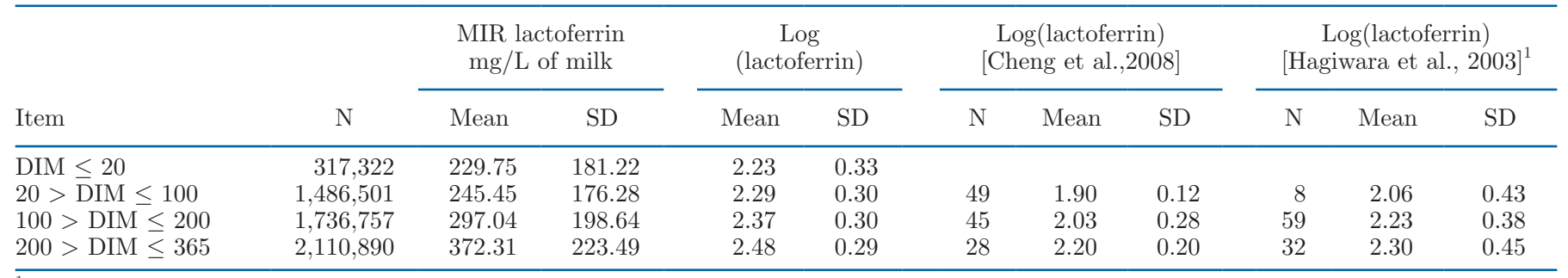

${ }^{1}$ The range of DIM was slightly different. 
Table 4. Evolution of lactoferrin content predicted by MIR following the milk yield ( $\mathrm{kg} /$ day) and comparison with the literature

\begin{tabular}{|c|c|c|c|c|c|c|c|c|}
\hline \multirow[b]{2}{*}{ Item } & \multirow[b]{2}{*}{$\mathrm{N}$} & \multicolumn{2}{|c|}{$\begin{array}{l}\text { MIR lactoferrin } \\
\mathrm{mg} / \mathrm{L} \text { of milk }\end{array}$} & \multicolumn{2}{|c|}{$\log$ (lactoferrin) } & \multicolumn{3}{|c|}{$\begin{array}{l}\log (\text { lactoferrin) } \\
\text { [Cheng et al.,2008] }\end{array}$} \\
\hline & & Mean & $\mathrm{SD}$ & Mean & $\mathrm{SD}$ & $\mathrm{N}$ & Mean & SD \\
\hline $20 \mathrm{~kg} \leq$ milk yield $<25 \mathrm{~kg}$ & $1,333,645$ & 308.94 & 204.31 & 2.39 & 0.30 & 34 & 1.99 & 0.23 \\
\hline $25 \mathrm{~kg}<$ milk yield $<30 \mathrm{~kg}$ & $1,201,798$ & 283.77 & 194.82 & 2.35 & 0.30 & 33 & 1.93 & 0.16 \\
\hline $30 \mathrm{~kg} \geq$ milk yield & $1,504,410$ & 254.63 & 181.96 & 2.30 & 0.30 & 19 & 1.89 & 0.18 \\
\hline
\end{tabular}

was already observed by Gaunt et al. (1980). The differences per lactation stages of the log-transformed LF contents obtained by predictions and found by Cheng et al. (2008) and Hagiwara et al. (2003) were very similar, (Table 3) even if the contents observed in this study were closer to the ones obtained by Hagiwara et al. (2003).

We also observed a negative correlation with milk yield $(-0.24)$, but a positive correlation with fat and protein contents (0.11 and 0.28 , respectively). Cheng et al. (2008) also found a strong positive relationship with protein $(r=0.48)$, but they mentioned that the correlation with fat content was not significantly different from 0 . However, the negative relationship between LF and milk yield was stronger for Cheng et al. (2008), where $r=-0.47$. The difference of LF after log-transformation, and observed by the level of milk yield, was similar even if the contents found in this study were higher (Table 4). As expected, positive correlations with predicted $\mathrm{LF}$ were observed for SCC and SCS $(0.21$ and 0.30 , respectively; $\mathrm{n}=5,477,197)$. Cheng et al. (2008) mentioned that the correlation between LF and SCC was not significantly different than 0 , but the correlation found by these authors for SCS $(r=0.37)$ was similar to the one estimated in the current study. The evolution of log-transformed predicted LF was also in agreement with the results found by Hagiwara et al. (2003) and Cheng et al. (2008), as seen in Table 5.

\section{CONCLUSIONS}

This study tried 4 examined machine learning algorithms to predict the daily content of LF in cow milk from milk MIR spectral data. It found, based on the validation prediction performances, that PLS, PLS + polynomial SVR, and PLS + ANN provided similar results, but that the model using PLS factors combined with an ANN was the best. This model was then applied to the Walloon milk recording spectral database to observe the relationships between predicted LF content and the main milk components as well as SCC and SCS, which were in line with the literature. However, the model still had some difficulties in predicting extremely high values. Indeed, the observed RMSE increased strongly once LF content exceeded $600 \mathrm{mg} / \mathrm{L}$ of milk; $12 \%$ of records coming from the Walloon dairy cow population reached this level of LF production. The inclusion of extreme values of milk LF content to the calibration set could help to improve the prediction models. We now have the possibility to directly predict the content of LF in the milk laboratory, allowing identification of those specific samples. Moreover, as the quantity of milk required for the ELISA analysis is quite low, the same sample as the one analyzed for the routine milk recording could be used. This could be an appropriate task to improve in the future of the ability of the ANN network to discriminate low and

Table 5. Evolution of lactoferrin content predicted by mid-infrared (MIR) spectrometry following the SCS ${ }^{1}$ and comparison with the literature

\begin{tabular}{|c|c|c|c|c|c|c|c|c|c|c|c|}
\hline \multirow[b]{2}{*}{ SCS } & \multirow[b]{2}{*}{$\mathrm{N}$} & \multicolumn{2}{|c|}{ MIR lactoferrin } & \multicolumn{2}{|c|}{ Log(lactoferrin) } & \multicolumn{3}{|c|}{$\begin{array}{l}\log (\text { lactoferrin }) \\
{[\text { Cheng et al.,2008] }}\end{array}$} & \multicolumn{3}{|c|}{$\begin{array}{c}\log \text { (lactoferrin) } \\
\text { [Hagiwara et al., 2003] }\end{array}$} \\
\hline & & Mean & SD & Mean & SD & $\mathrm{N}$ & Mean & SD & $\mathrm{N}$ & Mean & SD \\
\hline 0 & 163,716 & 236.23 & 166.91 & 2.28 & 0.29 & 12 & 1.91 & 0.14 & 36 & 2.18 & 0.19 \\
\hline 1 & 996,710 & 233.82 & 169.95 & 2.27 & 0.29 & 20 & 2.02 & 0.17 & 28 & 2.16 & 0.42 \\
\hline 2 & $1,365,672$ & 267.61 & 184.44 & 2.33 & 0.30 & 50 & 1.98 & 0.19 & 39 & 2.27 & 0.51 \\
\hline 3 & $1,164,258$ & 305.80 & 199.50 & 2.39 & 0.30 & 40 & 2.06 & 0.26 & & & \\
\hline 6 & 292,339 & 403.68 & 234.51 & 2.52 & 0.30 & 22 & 2.28 & 0.30 & & & \\
\hline 7 & 160,828 & 421.79 & 234.70 & 2.54 & 0.30 & & & & & & \\
\hline 8 & 83,575 & 461.05 & 235.88 & 2.59 & 0.28 & & & & & & \\
\hline 9 & 42,340 & 569.97 & 244.31 & 2.70 & 0.25 & & & & & & \\
\hline
\end{tabular}

${ }^{1} 10,557$ records were deleted because the SCS had a negative value. 
high LF samples. Until now, no implementation of this LF prediction has been done by DHI. However, there is an interest for them to use this molecule information to improve the detection of subclinical mastitis. The inclusion of the LF trait into breeding program to improve the cow robustness or the milk nutritional quality has not been investigated yet.

\section{ACKNOWLEDGMENTS}

This research received financial support from the European Commission, Directorate-General for Agriculture and Rural Development, under Grant Agreement 211708 and from the Commission of the European Communities, FP7, KBBE-2007-1. This paper does not necessarily reflect the view of these institutions and in no way anticipates the Commission's future policy in this area. The Ministry of Agriculture of the Walloon Region of Belgium [Service public de Wallonie, Direction générale de l'Agriculture, des Ressources naturelles et de l'Environnement, Direction de la Recherche] is acknowledged for their financial support through the project NovaUdderHealth (D31-1207). Scottish Agricultural College receives funding from the Scottish Government and they are acknowledged for funding the long-term selection experiment producing milk samples used in this analysis. The work of Sinead McParland was funded by a Science Foundation Ireland Starting Investigator Research Grant (18/SIRG/5562). The authors have not stated any conflicts of interest.

\section{REFERENCES}

Beck, M. W. 2018. NeuralNetTools: Visualization and analysis tools for neural networks. J. Stat. Softw. 85:1-20. https://doi.org/10 $.18637 /$ jss.v085.i11.

Chaneton, L., M. Bontá, M. Pol, L. Tirante, and L. E. Bussmann. 2013. Milk lactoferrin in heifers: Influence of health status and stage of lactation. J. Dairy Sci. 96:4977-4982. https://doi.org/10 .3168/jds.2012-6028.

Chaneton, L., L. Tirante, J. Maito, J. Chaves, and L. E. Bussmann. 2008. Relationship between milk lactoferrin and etiological agent in the mastitic bovine mammary gland. J. Dairy Sci. 91:18651873. https://doi.org/10.3168/jds.2007-0732.

Chen, P. W., and F. C. Mao. 2004. Detection of lactoferrin in bovine and goat milk by enzyme-linked immunosorbent assay. Yao Wu Shi Pin Fen Xi 12:133-139.

Cheng, J. B., J. Q. Wang, D. P. Bu, G. L. Liu, C. G. Zhang, H. Y. Wei, L. Y. Zhou, and J. Z. Wang. 2008. Factors Affecting the Lactoferrin Concentration in Bovine Milk. J. Dairy Sci. 91:970-976. https: //doi.org/10.3168/jds.2007-0689.

De Marchi, M., C. C. Fagan, C. P. O'Donnell, A. Cecchinato, R. Dal Zotto, M. Cassandro, M. Penasa, and G. Bittante. 2009. Prediction of coagulation properties, titratable acidity, and $\mathrm{pH}$ of bovine milk using mid-infrared spectroscopy. J. Dairy Sci. 92:423-432. https://doi.org/10.3168/jds.2008-1163.

Delhez, P., P. N. Ho, N. Gengler, H. Soyeurt, and J. E. Pryce. 2020. Diagnosing the pregnancy status of dairy cows: How useful is milk mid-infrared spectroscopy? J. Dairy Sci. 103:3264-3274. https:// doi.org/10.3168/jds.2019-17473.
Despagne, F., D. Luc Massart, and P. Chabot. 2000. Development of a robust calibration model for nonlinear in-line process data. Anal. Chem. 72:1657-1665. https://doi.org/10.1021/ac991076k.

Dórea, J. R. R., G. J. M. Rosa, K. A. Weld, and L. E. Armentano. 2018. Mining data from milk infrared spectroscopy to improve feed intake predictions in lactating dairy cows. J. Dairy Sci. 101:58785889. https://doi.org/10.3168/jds.2017-13997.

Finn, G. D., R. Lister, T. Szabo, D. Simonetta, H. Mulder, and R. Young. 1996. Neural networks applied to a large biological database to analyse dairy breeding patterns. Neural Comput. Appl. 4:237-253. https://doi.org/10.1007/BF01413822.

Gaunt, S. N., N. Raffio, E. T. Kingsbury, R. A. Damon Jr., W. H. Johnson, and B. A. Mitchell. 1980. Variation of lactoferrin and mastitis and their heritabilities. J. Dairy Sci. 63:1874-1880. https: //doi.org/10.3168/jds.S0022-0302(80)83154-7.

Giansanti, F., G. Panella, L. Leboffe, and G. Antonini. 2016. Lactoferrin from milk: Nutraceutical and pharmacological properties. Pharmaceuticals (Basel) 9:61. https://doi.org/10.3390/ph9040061.

Grelet, C., C. Bastin, M. Gelé, J. B. Davière, M. Johan, A. Werner, R. Reding, J. A. Fernandez Pierna, F. G. Colinet, P. Dardenne, N. Gengler, H. Soyeurt, and F. Dehareng. 2016. Development of Fourier transform mid-infrared calibrations to predict acetone, $\beta$-hydroxybutyrate, and citrate contents in bovine milk through a European dairy network. J. Dairy Sci. 99:4816-4825. https://doi .org/10.3168/jds.2015-10477.

Grelet, C., J. A. F. Pierna, P. Dardenne, H. Soyeurt, A. Vanlierde, F. Colinet, C. Bastin, N. Gengler, V. Baeten, and F. Dehareng. 2017. Standardization of milk mid-infrared spectrometers for the transfer and use of multiple models. J. Dairy Sci. 100:7910-7921. https://doi.org/10.3168/jds.2017-12720.

Grzesiak, W., P. Blaszczyk, and R. Lacroix. 2006. Methods of predicting milk yield in dairy cows-Predictive capabilities of Wood's lactation curve and artificial neural networks (ANNs). Comput. Electron. Agric. 54:69-83. https://doi.org/10.1016/j.compag.2006 .08 .004 .

Hagiwara, S-I., K. Kawai, A. Anri, and H. Nagahata. 2003. Lactoferrin concentrations in milk from normal and subclinical mastitic cows. J. Vet. Med. Sci. 65:319-323. https://doi.org/10.1292/jvms .65.319.

Hempstalk, K., S. McParland, and D. P. Berry. 2015. Machine learning algorithms for the prediction of conception success to a given insemination in lactating dairy cows. J. Dairy Sci. 98:5262-5273. https://doi.org/10.3168/jds.2014-8984.

Kawai, K., S. Hagiwara, A. Anri, and H. Nagahata. 1999. Lactoferrin concentration in milk of bovine clinical mastitis. Vet. Res. Commun. 23:391-398. https://doi.org/10.1023/A:1006347423426.

Król, J., Z. Litwińczuk, A. Brodziak, and J. Barłowska. 2010. Lactoferrin, lysozyme and immunoglobulin G content in milk of four breeds of cows managed under intensive production system. Pol. J. Vet. Sci. 13:357-361.

Kuhn, M. 2008. caret Package. J. Stat. Softw. 28:1-26.

Lê, S., J. Josse, and F. Husson. 2008. FactoMineR: An R package for multivariate analysis. J. Stat. Softw. 25:1-18. https://doi.org/10 .18637 /jss.v025.i01.

McCulloch, W. S., and W. Pitts. 1943. A logical calculus of the ideas imminent in nervous activity. Bull. Math. Biophys. 5:115-133. https://doi.org/10.1007/BF02478259.

Molenaar, A. J., Y. M. Kuys, S. R. Davis, R. J. Wilkins, P. E. Mead, and J. W. Tweedie. 1996. Elevation of lactoferrin gene expression in developing, ductal, resting regressing parenchymal epithelium of the ruminant mammary gland. J. Dairy Sci. 79:1198-1208. https:/ /doi.org/10.3168/jds.S0022-0302(96)76473-1.

Pralle, R. S., K. W. Weigel, and H. M. White. 2018. Predicting blood $\beta$-hydroxybutyrate using milk Fourier transform infrared spectrum, milk composition, and producer-reported variables with multiple linear regression, partial least squares regression, and artificial neural network. J. Dairy Sci. 101:4378-4387. https://doi .org/10.3168/jds.2017-14076.

Prekopcsak, Z., T. Henk, and C. Gaspar-Papanek. 2010. Cross-validation: The illusion of reliable performance estimation. Pages 1N6 in 
RCOMM RapidMiner Community Meeting and Conference. http: //prekopcsak.hu/papers/preko-2010-rcomm.pdf.

Soyeurt, H., C. Bastin, F. G. Colinet, V. M. R. Arnould, D. P. Berry, E. Wall, F. Dehareng, H. N. Nguyen, P. Dardenne, J. Schefers, J. Vandenplas, K. Weigel, M. Coffey, L. Thé Ron, J. Detilleux, E. Reding, N. Gengler, and S. McParland. 2012. Mid-infrared prediction of lactoferrin content in bovine milk: Potential indicator of mastitis. Animal 6:1830-1838. https://doi.org/10.1017/ S1751731112000791.

Soyeurt, H., F. G. Colinet, V. M. R. Arnould, P. Dardenne, C. Bertozzi, R. Renaville, D. Portetelle, and N. Gengler. 2007. Genetic variability of lactoferrin content estimated by mid-infrared spectrometry in bovine milk. J. Dairy Sci. 90:4443-4450. https://doi .org/10.3168/jds.2006-827.

Soyeurt, H., F. Dehareng, N. Gengler, S. McParland, E. Wall, D. P. Berry, M. Coffey, and P. Dardenne. 2011. Mid-infrared prediction of bovine milk fatty acids across multiple breeds, production systems, and countries. J. Dairy Sci. 94:1657-1667. https://doi.org/ 10.3168/jds.2010-3408.

Soyeurt, H., E. Froidmont, I. Dufrasne, D. Hailemariam, Z. Wang, C. Bertozzi, F. G. Colinet, F. Dehareng, and N. Gengler. 2019. Contribution of milk mid-infrared spectrum to improve the accuracy of test-day body weight predicted from stage, lactation number, month of test and milk yield. Livest. Sci. 227:82-89. https://doi .org/10.1016/j.livsci.2019.07.007.

Thissen, U., M. Pepers, B. Üstün, W. J. Melssen, and L. M. C. Buydens. 2004. Comparing support vector machines to PLS for spectral regression applications. Chemom. Intell. Lab. Syst. 73:169-179. https://doi.org/10.1016/j.chemolab.2004.01.002.

Vanlierde, A., M. L. Vanrobays, N. Gengler, P. Dardenne, E. Froidmont, H. Soyeurt, S. McParland, E. Lewis, M. H. Deighton, M Mathot, and F. Dehareng. 2016. Milk mid-infrared spectra enable prediction of lactation-stage-dependent methane emissions of dairy cattle within routine population-scale milk recording schemes. Anim. Prod. Sci. 56:258-264. https://doi.org/10.1071/AN15590.

Wakabayashi, H., K. Yamauchi, and M. Takase. 2006. Lactoferrin research, technology and applications. Int. Dairy J. 16:1241-1251. https://doi.org/10.1016/j.idairyj.2006.06.013.

Wang, Q., and H. Bovenhuis. 2019. Validation strategy can result in an overoptimistic view of the ability of milk infrared spectra to predict methane emission of dairy cattle. J. Dairy Sci. 102:6288-6295. https://doi.org/10.3168/jds.2018-15684.

Whitfield, R. G., M. E. Gerger, and R. L. Sharp. 1987. Near-infrared spectrum qualification via Mahalanobis distance determination. Appl. Spectrosc. 41:1204-1213. https://doi.org/10.1366/ 0003702874447572

Yang, X. Z., R. Lacroix, and K. M. Wade. 2000. Investigation into the production and conformation traits associated with clinical mastitis using artificial neural networks. Can. J. Anim. Sci. 80:415-426. https://doi.org/10.4141/A98-100.

\section{ORCIDS}

H. Soyeurt ๑ https://orcid.org/0000-0001-9883-9047

C. Grelet 10 https://orcid.org/0000-0003-3313-485X

S. McParland @ https://orcid.org/0000-0003-3320-2330

M. Calmels @ https://orcid.org/0000-0001-7243-7920

M. Coffey ๑ https://orcid.org/0000-0003-4890-6218

A. Tedde ๑ https://orcid.org/0000-0002-4882-3386

P. Delhez ㄴ https://orcid.org/0000-0002-2699-1135

F. Dehareng () https://orcid.org/0000-0002-6733-4334

N. Gengler () https://orcid.org/0000-0002-5981-5509 